Homology, Homotopy and Applications, vol.17(2), 2015, pp.191-204

\title{
THE HAMMOCK LOCALIZATION PRESERVES HOMOTOPIES
}

\author{
ORIOL RAVENTÓS
}

(communicated by Brooke Shipley)

\begin{abstract}
The hammock localization provides a model for a homotopy function complex in any Quillen model category. We prove that a homotopy between a pair of morphisms induces a homotopy between the maps induced by taking the hammock localization. We also show that, under Vopěnka's principle, every homotopy idempotent functor in a cofibrantly generated model category is determined by simplicial orthogonality with respect to a set of morphisms. Finally, we give a new proof of the fact that left Bousfield localizations with respect to a class of morphisms always exist in any left proper combinatorial model category under Vopěnka's principle.
\end{abstract}

\section{Introduction}

The hammock localization was introduced by Dwyer and Kan in a series of articles $[\mathbf{D K 8 0 a}, \mathbf{D K} 80 \mathrm{c}, \mathbf{D K} 80 \mathrm{~b}]$. Given a category $\mathcal{C}$ with a fixed class of morphisms $\mathcal{W}$, the hammock localization $\mathcal{L}^{H} \mathcal{C}$ is a simplicial category such that $\pi_{0}\left(\mathcal{L}^{H} \mathcal{C}(X, Y)\right)$ is the set of morphisms from $X$ to $Y$ in the category obtained by inverting the morphisms in $\mathcal{W}$ for every pair of objects $X$ and $Y$ in $\mathcal{C}$. In the case that $\mathcal{C}$ is a model category and $\mathcal{W}$ is its class of weak equivalences, then $\pi_{0}\left(\mathcal{L}^{H} \mathcal{C}(X, Y)\right)$ is in natural bijection with the set of homotopy classes of morphisms $[X, Y]$ and, as a bifunctor, $\mathcal{L}^{H} \mathcal{C}(-,-)$ sends weak equivalences to weak homotopy equivalences. Hence, $\mathcal{L}^{H} \mathcal{C}(-,-)$ defines a homotopy function complex on $\mathcal{C}$. Moreover, if $\mathcal{C}$ is a simplicial model category, with simplicial mapping space $\operatorname{Map}(-,-)$, then $\mathcal{L}^{H} \mathcal{C}(X, Y) \simeq$ $\operatorname{Map}\left(X^{c}, Y^{f}\right)$, where $X^{c}$ is a cofibrant replacement of $X$ and $Y^{f}$ is a fibrant replacement of $Y$.

In Theorem 3.1 we prove that $\mathcal{L}^{H} \mathcal{C}(-,-)$ sends left or right homotopies to simplicial homotopies. This is applied in Section 4 to study homotopy idempotent functors. We recall that a (coaugmented) homotopy idempotent functor on a model category $\mathcal{C}$ is a functor $L: \mathcal{C} \rightarrow \mathcal{C}$ together with a natural transformation $\ell: 1 \rightarrow L$ that induces

The author is supported by the grant SFB 1085 "Higher invariants" funded by the German Research Foundation, the project CZ.1.07/2.3.00/20.0003 of the Operational Programme Education for Competitiveness of the Ministry of Education, Youth and Sports of the Czech Republic, the Spanish Ministry of Science and Innovation under the grants MTM2010-15831 and MTM2013-42178-P, and the Government of Catalonia under the grant SGR-119-2009.

Received December 4, 2014, revised May 12, 2015; published on November 18, 2015. 2010 Mathematics Subject Classification: 55U35, 55P60, $18 \mathrm{C} 35$.

Key words and phrases: model category, homotopy function complex, localization, homotopy algebra. Article available at http://dx.doi.org/10.4310/HHA.2015.v17.n2.a10

Copyright (C) 2015, International Press. Permission to copy for private use granted. 
a localization, i.e., a left adjoint of the inclusion of a reflective subcategory, in the homotopy category. An object $X$ is $L$-local if it is weakly equivalent to an object of the form $L Y$ for some $Y$, and a morphism $f$ is an $L$-equivalence if $L f$ is a weak equivalence. We prove in Proposition 4.3 that, in any model category, $L$-local objects and $L$-equivalences are simplicially orthogonal with respect to $\mathcal{L}^{H} \mathcal{C}(-,-)$. The first result of this kind was obtained by Dror Fajoun in [Far96b] in the category of spaces, and later it was extended to other contexts (cf. $[\mathbf{C S S 0 5 , ~ C C 0 6}])$. If we assume a certain large cardinal axiom, called Vopěnka's principle, we prove in Corollary 4.10 that for each homotopy idempotent functor $(L, \ell)$ in any cofibrantly generated model category, the class of $L$-local objects correspond to the class of objects that are simplicially orthogonal to just a set of morphisms. This result extends a previous result in [CC06, Theorem 2.3] for simplicial combinatorial model categories to all cofibrantly generated model categories. In the same spirit, we extend in Theorem 4.12 the analogous result for augmented homotopy idempotent functors [Cho07, Theorem 2.1].

It was proved in [RT03, Theorem 2.3] that, under Vopěnka's principle, left Bousfield localizations with respect to a class of morphisms exist in any left proper combinatorial model category. We give a new proof of this fact in Corollary 4.6. The proof can be easily modified to give the analogous result for right Bousfield localizations, as we state in Corollary 4.11. This last result extends a previous result in [Cho07, Theorem 1.4] for simplicial combinatorial model categories to all combinatorial model categories.

The hammock localization $\mathcal{L}^{H}$ can be extended to a functor from the category of small categories with weak equivalences to the category of small simplicial categories

$$
\mathcal{L}^{H}: \text { wCat } \longrightarrow \text { sCat }
$$

as we make precise in Section 3. We prove that $\mathcal{L}^{H}$ can be extended so as to send natural transformations to simplicial natural transformations up to homotopy in Theorem 3.2. This result is used in a joint paper of the author with Casacuberta and Tonks $[\mathbf{C R T}]$ to study homotopy algebra structures preserved by localizations.

\section{Acknowledgments}

We want to thank Ilias Amrani, John Bourke, Carles Casacuberta, Alexandru Stanculescu, Javier Gutiérrez, George Raptis, and Andrew Tonks for useful discussions during the preparation of this article.

\section{The hammock localization}

The hammock localization defines one model for the homotopy function complex of a model category. It was introduced by Dwyer and Kan in a series of articles [DK80a, DK80c, DK80b]. We will summarize some of their results following the more recent exposition contained in [DHKS04, Chapters 34 and 35].

A category with weak equivalences is a pair $(\mathcal{C}, \mathcal{W})$ with $\mathcal{C}$ a category and $\mathcal{W}$ a fixed class of morphisms in $\mathcal{C}$ that contains all identities. The morphisms in $\mathcal{W}$ are called weak equivalences. Assume (just for the moment) that $\mathcal{C}$ is small. For every pair of objects $X$ and $Y$ in $\mathcal{C}$, and every odd natural number $n$, we define a category 
$\mathcal{L}_{n}^{H} \mathcal{C}(X, Y)$ with objects being strings of $n$ morphisms on $\mathcal{C}$ in alternating directions

$$
C_{0} \stackrel{d_{0}}{\longleftarrow} C_{1} \stackrel{d_{1}}{\longrightarrow} \ldots \stackrel{d_{n-2}}{\longrightarrow} C_{n-1} \stackrel{d_{n-1}}{\longleftarrow} C_{n},
$$

with $X=C_{0}, Y=C_{n}$ and the arrows pointing to the left being weak equivalences. A morphism is a commutative diagram of the form

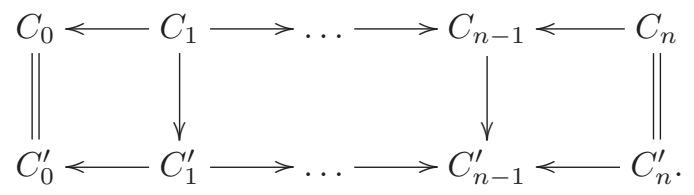

The hammock localization of $(\mathcal{C}, \mathcal{W})$ is a simplicial category (meaning simplicially enriched) $\mathcal{L}^{H} \mathcal{C}$ with the same objects as $\mathcal{C}$ and, for every pair of objects $X$ and $Y$, a simplicial set

$$
\mathcal{L}^{H} \mathcal{C}(X, Y)=\underset{k}{\operatorname{colim}} N \mathcal{L}_{2 k+1}^{H} \mathcal{C}(X, Y),
$$

where the sequential colimit (that is also a homotopy colimit) is taken over the nerve of the embedding functors that send an object like $(1)$ in $\mathcal{L}_{n}^{H} \mathcal{C}(X, Y)$ to

$$
X=C_{0} \longleftarrow C_{1} \stackrel{i d}{\longrightarrow} C_{1} \stackrel{i d}{\longleftarrow} C_{1} \longrightarrow \ldots \longrightarrow C_{n-1} \longleftarrow C_{n}=Y
$$

in $\mathcal{L}_{n+2}^{H} \mathcal{C}(X, Y)$. The composition in $\mathcal{L}^{H} \mathcal{C}$ is given by concatenation. More precisely, given an object

$$
X<C_{1} \longrightarrow \ldots \longrightarrow C_{n-1} \stackrel{d_{n-1}}{\longleftarrow} Y
$$

in $\mathcal{L}_{n}^{H} \mathcal{C}(X, Y)$ and an object

$$
Y \stackrel{d_{0}^{\prime}}{\longleftarrow} C_{1}^{\prime} \longrightarrow \ldots \longrightarrow C_{n-1}^{\prime} \longleftarrow Z
$$

in $\mathcal{L}_{n}^{H} \mathcal{C}(Y, Z)$, their composition is the object

$$
X \longleftarrow C_{1} \longrightarrow \ldots \longrightarrow C_{n-1} \stackrel{d_{n-1} \circ d_{0}^{\prime}}{\longleftarrow} C_{1}^{\prime} \longrightarrow \ldots \longrightarrow C_{n-1}^{\prime} \longleftarrow Z
$$

in $\mathcal{L}_{2 n-1}^{H} \mathcal{C}(X, Z)$. Hence, for every triple of objects $X, Y$, and $Z$ in $\mathcal{C}$, we define a simplicial composition $\mathcal{L}^{H} \mathcal{C}(X, Y) \times \mathcal{L}^{H} \mathcal{C}(Y, Z) \rightarrow \mathcal{L}^{H} \mathcal{C}(X, Z)$ as

$$
\operatorname{colim}_{k} N\left(\mathcal{L}_{2 k+1}^{H} \mathcal{C}(X, Y) \times \mathcal{L}_{2 k+1}^{H} \mathcal{C}(Y, Z) \rightarrow \mathcal{L}_{4 k+1}^{H} \mathcal{C}(X, Z)\right)
$$

Here we use the well-known facts that filtered colimits commute with finite limits, that nerves commute with products, and that the category of simplicial sets is a cartesian closed model category. The identity of $X$ is determined by fixing, for each $n$, the object in $\mathcal{L}_{n}^{H}(X, X)$ with only identity arrows. The associativity and identity properties can be checked levelwise.

Remark 2.1. The hammock localization was originally defined using a colimit over all natural numbers (cf. [DK80b]). We restrict to odd natural numbers because in this case the morphisms in the extremes are always going backwards and then we do not need to distinguish two cases in every proof. It can be seen that both definitions 
coincide using a cofinality argument, as proved in [DHKS04, Chapter 34]. It is also worth mentioning that if $\mathcal{C}$ is a model category and $\mathcal{W}$ is its class of weak equivalences, then $\mathcal{L}^{H} \mathcal{C}(X, Y) \simeq \mathcal{L}_{3}^{H} \mathcal{C}(X, Y)$ for every pair of objects $X$ and $Y$ (cf. [DK80b]). Although working with $\mathcal{L}_{3}^{H}$ has certain advantages, for the purposes of this article it is more convenient to work with $\mathcal{L}^{H}$ even in the case of model categories.

Remark 2.2. We recall that the nerve of a category $\mathcal{D}$ is the simplicial set with $n$-simplices $(N \mathcal{D})_{n}=\operatorname{Cat}([n], \mathcal{D})$ and that this defines a fully faithful functor from Cat to the category of simplicial sets. We will often use the fact that natural transformations induce homotopies after taking nerves [Qui73, Section 1, Proposition 2]. In particular, adjunctions induce homotopy equivalences. It is also useful to know that, for any pair of objects $X$ and $Y, \mathcal{L}^{H} \mathcal{C}(X, Y)$ is weakly equivalent to the nerve of the Grothendieck construction on the diagram defining the sequential colimit, as observed in [DHKS04, Proposition 35.7].

Definition 2.3. Let wCat denote the category of small categories with weak equivalences and morphisms being the functors that preserve weak equivalences. Then there is a functor

$$
\mathcal{L}^{H}: \text { wCat } \longrightarrow \text { sCat }
$$

where sCat is the category of small simplicial categories. The image of a morphism $F: \mathcal{C} \rightarrow \mathcal{C}^{\prime}$ in wCat is defined levelwise, i.e., each object $X$ in $\mathcal{L}^{H} \mathcal{C}$ is sent to $F X$ in $\mathcal{L}^{H} \mathcal{C}^{\prime}$ and, for each pair of objects $X$ and $Y$ in $\mathcal{C}$, we define a simplicial map $\mathcal{L}^{H} \mathcal{C}(X, Y) \rightarrow \mathcal{L}^{H} \mathcal{C}^{\prime}(F X, F Y)$ determined by sending an object like (1) in $\mathcal{L}_{n}^{H} \mathcal{C}(X, Y)$ to

$$
F X \stackrel{F d_{0}}{\longleftarrow} F C_{1} \stackrel{F d_{1}}{\longrightarrow} \ldots \stackrel{F d_{n-2}}{\longrightarrow} F C_{n-1} \stackrel{F d_{n-1}}{\longleftarrow} F Y
$$

in $\mathcal{L}_{n}^{H} \mathcal{C}^{\prime}(F X, F Y)$.

Notice that for every morphism $f: A \rightarrow B$ in a category $\mathcal{C}$ there are induced maps of simplicial sets

$$
f^{*}: \mathcal{L}^{H} \mathcal{C}(B, Y) \longrightarrow \mathcal{L}^{H} \mathcal{C}(A, Y) \text { and } f_{*}: \mathcal{L}^{H} \mathcal{C}(X, A) \longrightarrow \mathcal{L}^{H} \mathcal{C}(X, B)
$$

for all $X$ and $Y$ in $\mathcal{C}$. They are defined just using the composition in $\mathcal{L}^{H} \mathcal{C}$ and fixing one of the variables as the diagram

$$
A<\stackrel{i d}{\stackrel{f}{\longrightarrow}} B \stackrel{i d}{\longrightarrow} B
$$

in $\mathcal{L}_{3}^{H} \mathcal{C}(A, B)$. To be more precise, $f^{*}$ is induced by the functors $f_{n}^{*}$ that send an object like (1) in $\mathcal{L}_{n}^{H} \mathcal{C}(B, Y)$ to

$$
A<i d) \stackrel{f}{\longrightarrow} B \ll C_{1} \longrightarrow \ldots \longrightarrow C_{n-1} \longleftarrow Y
$$

in $\mathcal{L}_{n+2}^{H} \mathcal{C}(A, Y)$ for every odd natural number $n$. If $f$ is a weak equivalence, then $f^{*}$ is a weak homotopy equivalence and a homotopy inverse is given by the functors that send an object like $(1)$ in $\mathcal{L}_{n}^{H} \mathcal{C}(A, Y)$ to

$$
B \stackrel{f}{\longleftarrow} A \stackrel{i d}{\longrightarrow} A \longleftarrow C_{1} \longrightarrow \ldots \longrightarrow C_{n-1} \longleftarrow Y
$$

in $\mathcal{L}_{n+2}^{H} \mathcal{C}(B, Y)$ for every odd natural number $n$. Indeed, if $f$ is a weak equivalence, 
then $f_{n}^{*}$ is an equivalence of categories for each $n$. The map $f_{*}$ is defined similarly.

If $\mathcal{C}$ is a model category, we will let $\mathcal{W}$ be exactly the class of weak equivalences in $\mathcal{C}$. In this case, $\pi_{0}\left(\mathcal{L}^{H} \mathcal{C}(X, Y)\right) \cong \operatorname{Ho}(\mathcal{C})(X, Y)$ and $\mathcal{L}^{H} \mathcal{C}(X, Y)$ defines a homotopy function complex (or homotopy mapping space) for $\mathcal{C}$ (cf. [Hir03, Chapter 17]).

We would like to apply the hammock localization not only to small categories. This has some technical set theoretical issues that can be nicely handled using the axiomatization of universes. We refer to [DHKS04, Section 32] for a detailed explanation.

\section{A property of the hammock localization}

The following result asserts that the hammock localization respects homotopies. For the basic properties of homotopies in model categories, we refer to [Hir03, Chapter 7]. As usual, by simplicial homotopy in a simplicial model category we mean the equivalence relation generated by the strict homotopies.

Theorem 3.1. Let $\mathcal{C}$ be a model category, and let $f, g: A \rightarrow B$ be a pair of left or right homotopic morphisms in $\mathcal{C}$. Then the induced maps $f_{*}, g_{*}: \mathcal{L}^{H} \mathcal{C}(X, A) \rightarrow \mathcal{L}^{H} \mathcal{C}(X, B)$ are simplicially homotopic for all $X$ in $\mathcal{C}$ and $f^{*}, g^{*}: \mathcal{L}^{H} \mathcal{C}(B, Y) \rightarrow \mathcal{L}^{H} \mathcal{C}(A, Y)$ are simplicially homotopic maps for all $Y$ in $\mathcal{C}$.

Proof. Assume that $f$ and $g$ are left homotopic. Fix a cylinder object

$$
A \amalg A \stackrel{i_{0} \amalg i_{1}}{\longrightarrow} \operatorname{Cyl}(A) \stackrel{p}{\longrightarrow} A,
$$

where $p \circ i_{0}=p \circ i_{1}=i d$ and $i_{0}, i_{1}$, and $p$ are weak equivalences. Let $H: \operatorname{Cyl}(A) \rightarrow B$ be a left homotopy between $f$ and $g$. Thus, $H \circ i_{0}=f$ and $H \circ i_{1}=g$.

Let $\tilde{H}: \mathcal{L}^{H} \mathcal{C}(X, A) \rightarrow \mathcal{L}^{H} \mathcal{C}(X, B)$ be the map induced by the functors $\tilde{H}^{n}$ that send an object like $(1)$ in $\mathcal{L}^{H} \mathcal{C}_{n}(X, A)$ to

$$
X \ll C_{1} \longrightarrow \ldots \longrightarrow C_{n-1} \stackrel{d_{n-1} \circ p}{\longleftarrow} \mathrm{Cyl}(A) \stackrel{H}{\longrightarrow} B<\stackrel{i d}{\longleftarrow} B
$$

in $\mathcal{L}^{H} \mathcal{C}_{n+2}(X, B)$. Hence, the commutative diagram

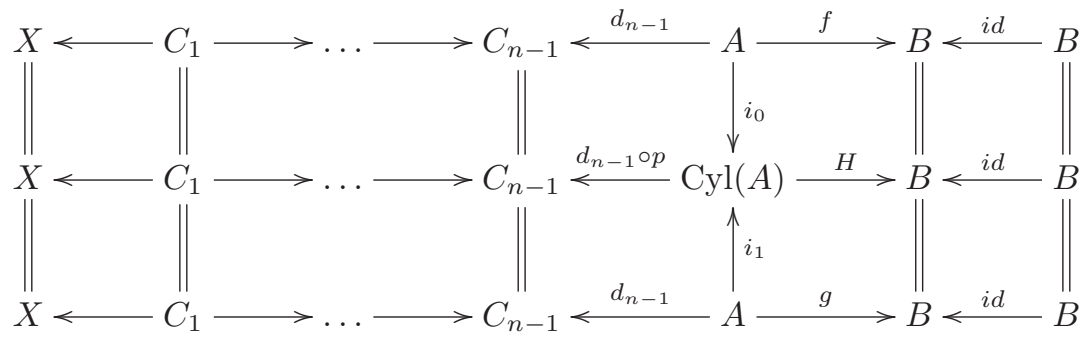

determines a zig-zag of natural transformations

$$
f_{*}^{n} \stackrel{\phi^{n}}{\longrightarrow} \tilde{H}^{n} \stackrel{\psi^{n}}{\longleftarrow} g_{*}^{n}
$$

between functors from $\mathcal{L}^{H} \mathcal{C}_{n}(X, A)$ to $\mathcal{L}^{H} \mathcal{C}_{n+2}(X, B)$ for each odd natural number $n$, 
which are compatible with the inclusions

$$
\mathcal{L}^{H} \mathcal{C}_{n}(X, A) \rightarrow \mathcal{L}^{H} \mathcal{C}_{n+2}(X, A) .
$$

Because $\tilde{H}=\operatorname{colim}_{k} N \tilde{H}^{2 k+1}$, the nerve functor sends natural transformations to simplicial homotopies, and $\phi^{n}$ and $\psi^{n}$ are compatible with the colimit, we have an induced zig-zag of homotopies of simplicial sets $f_{*} \simeq \tilde{H} \simeq g_{*}$.

It $f$ and $g$ are right homotopic, then the statement can be proved similarly using path objects.

The following result describes the image of a natural transformation under the hammock localization functor.

Theorem 3.2. Let $F, G: \mathcal{C} \rightarrow \mathcal{D}$ be a pair of functors in $\mathbf{w C a t}$ together with a natural transformation $\eta: F \rightarrow G$. There is a homotopy $\mathcal{L}^{H} \eta(X, Y)$ from $\eta_{Y *} \circ \mathcal{L}^{H} F(X, Y)$ to $\eta_{X}^{*} \circ \mathcal{L}^{H} G(X, Y)$ for each pair of objects $X$ and $Y$ in $\mathcal{C}$ :

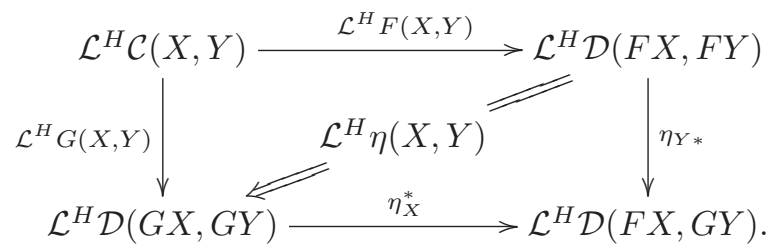

Notice that $\mathcal{L}^{H}$ fails to be a strict 2-functor in general. To be so, $\mathcal{L}^{H} \eta$ would have to define a simplicially enriched natural transformation, which exactly means that $\mathcal{L}^{H} \eta(X, Y)$ would have to be the identity for each pair of objects $X$ and $Y$ (cf. [Kel82, Section 1.2]). Nevertheless, Theorem 3.2 has already some applications. For instance, it is used in [CRT] to study homotopy algebras over monads in the homotopy category. Examples of such algebras include homotopy associative $H$-spaces or homotopy module spectra.

In the proof of Theorem 3.2, we will need the following observation that we state without a proof.

Lemma 3.3. Let $\mathcal{C}$ be a category in wCat. For any pair of objects $X$ and $Y$ in $\mathcal{C}$, the inclusion $\mathcal{L}_{n}^{H} \mathcal{C}(X, Y) \hookrightarrow \mathcal{L}_{n+2}^{H} \mathcal{C}(X, Y)$ in (2) that consists in inserting two consecutive identity morphisms in $C_{1}$ is related by a zig-zag of natural transformations to the inclusion defined by inserting two consecutive identity morphisms in $C_{i}$ for any $0 \leqslant i \leqslant n$.

Proof of Theorem 3.2. Fix an object like $(1)$ in $\mathcal{L}_{n}^{H} \mathcal{C}(X, Y)$. Then we can describe the homotopy $\mathcal{L}^{H} \eta(X, Y)$ by the natural transformation defined by the morphisms

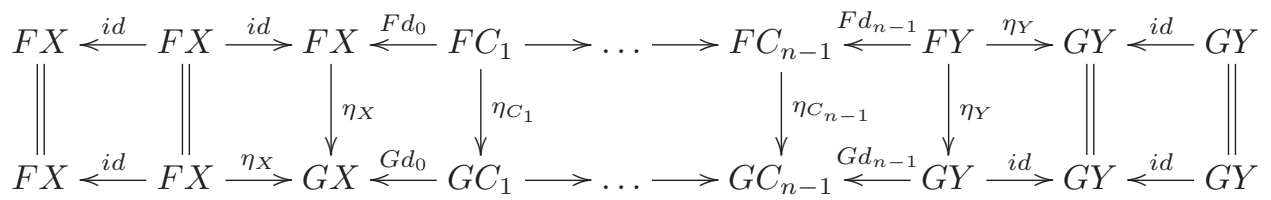

in $\mathcal{L}_{n+4}^{H} \mathcal{D}(F X, G Y)$ for each odd number $n$. It is a consequence of Lemma 3.3 that the two identities we artificially introduced at the beginning of the first row will have 
no effect after we take the colimit over $n$ to produce $\eta_{Y *} \circ \mathcal{L}^{H} F(X, Y)$. The same applies to the two identities at the end of the second row.

\section{Homotopy idempotent functors}

We next define an analogue of the notion of idempotent functor (cf. [Bor94, Section 4.2]), in the context of model categories following [CSS05].

Definition 4.1. Let $\mathcal{M}$ be a model category. A functor $L: \mathcal{M} \rightarrow \mathcal{M}$ together with a natural transformation $\ell: 1 \rightarrow L$ is called (coaugmented) homotopy idempotent if $L$ sends weak equivalences to weak equivalences and the natural morphisms $\ell_{L X}$ and $L \ell_{X}: L X \rightarrow L L X$ are equal in the homotopy category $\operatorname{Ho}(\mathcal{M})$ and both are weak equivalences for every object $X$ in $\mathcal{M}$.

There is a notion of augmented homotopy idempotent functor, also called cellularization. All results in this section have analogues for the augmented case, and the proofs can be easily transferred. At the end of the section, we will state the analogues of the two main results.

Given a homotopy idempotent functor $(L, \ell)$, a morphism $f$ in $\mathcal{M}$ is called an $L$-equivalence if $L f$ is a weak equivalence, and a fibrant object $X$ in $\mathcal{M}$ is called $L$-local if $X \simeq L Y$ for some $Y$ in $\mathcal{M}$. The class of $L$-equivalences and $L$-local objects determine each other by orthogonality in the homotopy category $\operatorname{Ho}(\mathcal{M})$. This means that a morphism $g: X \rightarrow Y$ is an $L$-equivalence if and only if the morphism

$$
g^{*}:[Y, Z] \stackrel{\cong}{\longrightarrow}[X, Z]
$$

is an isomorphism for every $L$-local object $Z$, and a fibrant object $Z$ is $L$-local if and only if $g^{*}$ is an isomorphism for all $L$-equivalences $g$ (cf. [Ada75, Proposition 2.10]).

We will prove in Proposition 4.3 that $L$-equivalences and $L$-local objects are also simplicially orthogonal in the model category. Let us explain what this means. Fix a homotopy function complex map $(-,-)$ in a model category $\mathcal{M}$, and let $\mathcal{S}$ be any class of morphisms in $\mathcal{M}$. A fibrant object $X$ in $\mathcal{M}$ is called $\mathcal{S}$-local if, for every morphism $f: A \rightarrow B$ in $\mathcal{S}$, the induced map of homotopy function complexes

$$
f^{*}: \operatorname{map}(B, X) \longrightarrow \operatorname{map}(A, X)
$$

is a weak homotopy equivalence. We denote by $\mathcal{S}^{h \perp}$ the class of $\mathcal{S}$-local objects and we call it the simplicial orthogonal complement of $\mathcal{S}$. Similarly, for any class of objects $\mathcal{D}$ in $\mathcal{M}$, a morphism $f: A \rightarrow B$ is called a $\mathcal{D}$-equivalence if, for every $X \in \mathcal{D}, f^{*}$ is

a weak homotopy equivalence. By an abuse of notation, we also denote by $\mathcal{D}^{h \perp}$ the class of $\mathcal{D}$-equivalences and we call it the simplicial orthogonal complement of $\mathcal{D}$.

It is important to notice that these definitions do not depend on the choice of homotopy function complex [Hir03, Proposition 17.8.2]. We fix $\operatorname{map}(-,-)$ to be $\mathcal{L}^{H} \mathcal{C}(-,-)$.

Recall from [Hir03, Definition 3.3.1] that the left Bousfield localization with respect to a class of morphisms $\mathcal{S}$ on a model category $\mathcal{M}$ (if it exists) is a new model category structure $L_{\mathcal{S}} \mathcal{M}$ on the same underlying category $\mathcal{M}$ with the same cofibrations and the weak equivalences being the $\mathcal{S}^{h \perp}$-equivalences. In particular, if we consider the fibrant replacement functor in $L_{\mathcal{S}} \mathcal{M}$, then it defines a homotopy 
idempotent functor on $\mathcal{M}$. We will show that, if we assume that Vopěnka's principle holds, then in any cofibrantly generated model category a homotopy idempotent functor has the same local objects as a left Bousfield localization with respect to a set of morphisms.

Lemma 4.2. Let $\mathcal{M}$ be a model category, and let $(L, \ell)$ be a homotopy idempotent functor on $\mathcal{M}$. For every pair of objects $X$ and $Y$,

1. the map $\mathcal{L}^{H} L(X, L Y): \operatorname{map}(X, L Y) \rightarrow \operatorname{map}(L X, L L Y)$ is a simplicial homotopy equivalence, and

2. the map $\ell_{X}^{*}: \operatorname{map}(L X, L Y) \rightarrow \operatorname{map}(X, L Y)$ is also a simplicial homotopy equivalence.

Proof. For the first part, we let $h: \operatorname{map}(L X, L L Y) \rightarrow \operatorname{map}(X, L Y)$ be the map induced by the functors $h^{n}$ that send an object like $(1)$ in $\mathcal{L}_{n}^{H} \mathcal{C}(L X, L L Y)$ to

$$
X \longleftarrow \stackrel{i d}{\longleftarrow} X \stackrel{\ell_{X}}{\longrightarrow} L X \longleftarrow C_{1} \longrightarrow \ldots \longrightarrow C_{n-1} \stackrel{d_{n-1} \circ \ell_{L Y}}{\longrightarrow} L Y
$$

in $\mathcal{L}_{n+2}^{H} \mathcal{C}(X, L Y)$ for every odd natural number $n$. The homotopy from the identity (see Lemma 3.3) to $h \circ \mathcal{L}^{H} L(X, L Y)$ is determined by the commutative diagram

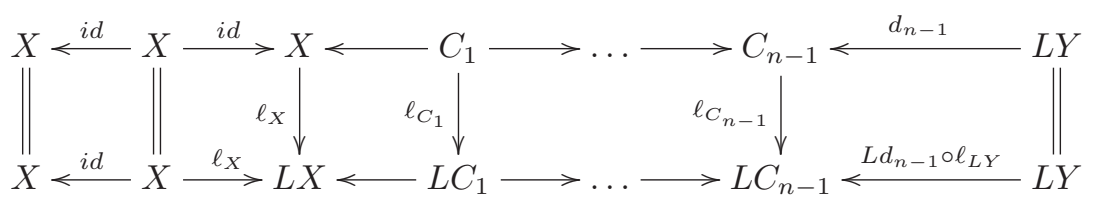

in $\mathcal{L}_{n+2}^{H} \mathcal{C}(X, L Y)$ for every odd natural number $n$. We will now define a zig-zag of homotopies between the identity and $\mathcal{L}^{H} L(X, L Y) \circ h$ induced by a zig-zag of natural transformations

$$
i d^{n} \longrightarrow \tilde{H}^{n} \longleftarrow \mathcal{L}_{n}^{H} L \circ h^{n}
$$

that are compatible with the inclusions

$$
\mathcal{L}_{n}^{H} \mathcal{C}(L X, L L Y) \longrightarrow \mathcal{L}_{n+2}^{H} \mathcal{C}(L X, L L Y) .
$$

Since $\operatorname{map}(-,-)$ is homotopy invariant, we can assume that $L X, L L X, L L Y$, and $L L L Y$ are fibrant and cofibrant. Hence, there are two cylinder objects

$$
\begin{gathered}
L X \amalg L X \stackrel{i_{0} \amalg i_{1}}{\longrightarrow} \operatorname{Cyl}(L X) \stackrel{p}{\longrightarrow} L X \quad \text { and } \\
L L Y \amalg L L Y \stackrel{i_{0}^{\prime} \amalg i_{1}^{\prime}}{\longrightarrow} \operatorname{Cyl}(L L Y) \stackrel{p^{\prime}}{\longrightarrow} L L Y,
\end{gathered}
$$

a left homotopy $H: \operatorname{Cyl}(L X) \rightarrow L L X$ between $H \circ i_{0}=L \ell_{X}$ and $H \circ i_{1}=\ell_{L X}$, and a left homotopy $H^{\prime}: \operatorname{Cyl}(L L Y) \rightarrow L L L Y$ between $H^{\prime} \circ i_{0}^{\prime}=L \ell_{L Y}$ and $H^{\prime} \circ i_{1}^{\prime}=\ell_{L L Y}$ (notice that $H^{\prime}$ is forced to be a weak equivalence by the two-out-of-three property). Let $\tilde{H}^{n}$ be the functor that sends an object like (1) in $\mathcal{L}_{n}^{H} \mathcal{C}(L X, L L Y)$ to

$$
L X \stackrel{p}{\leftarrow} \mathrm{Cyl}(L X) \stackrel{H}{\rightarrow} L L X \stackrel{L d_{0}}{\longleftarrow} L C_{1} \ldots L C_{n-1} \stackrel{L d_{n-1} \circ H^{\prime}}{\longleftarrow} \mathrm{Cyl}(L L Y) \stackrel{p^{\prime}}{\longrightarrow} L L Y \stackrel{i d}{\longleftarrow} L L Y
$$


in $\mathcal{L}_{n+4}^{H} \mathcal{C}(L X, L L X)$. The diagram

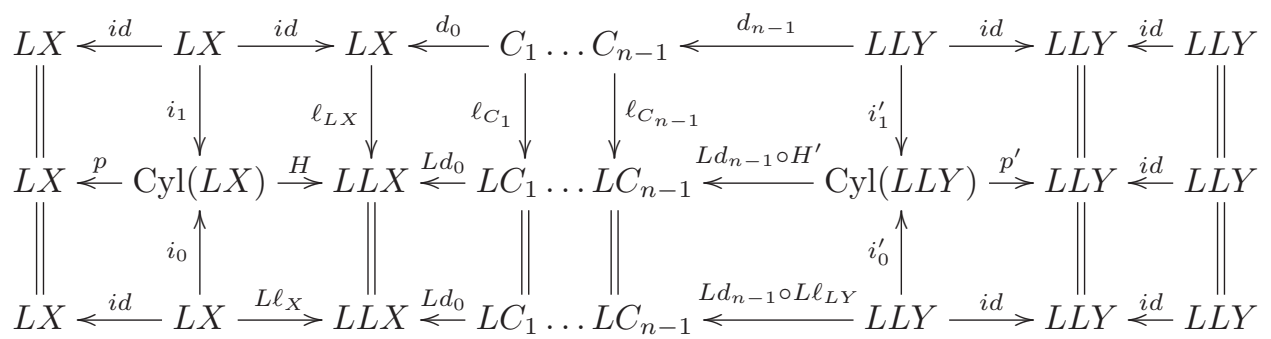

in $\mathcal{L}_{n+4}^{H} \mathcal{C}(L X, L L Y)$ defines the zig-zag of natural transformations (3) inducing the homotopy equivalence between $i d$ and $\mathcal{L}^{H} L \circ h$.

The second part of the statement follows from Theorem 3.2, because $\ell$ induces a homotopy $\ell_{L Y *} \simeq \ell_{X}^{*} \circ \mathcal{L}^{H} L(X, L Y)$, and $\ell_{L Y *}$ and $\mathcal{L}^{H} L(X, L Y)$ are weak homotopy equivalences.

Proposition 4.3. Let $\mathcal{M}$ be a model category, and let $(L, \ell)$ be a homotopy idempotent functor on $\mathcal{M}$. Then the class of $L$-equivalences coincides with the simplicial orthogonal complement of the class of L-local objects.

Proof. We first prove that $L$-local objects are simplicially orthogonal to $L$-equivalences: Fix an object $L Y$ and a morphism $f: A \rightarrow B$ such that $L f$ is a weak equivalence. We want to prove that $\operatorname{map}(f, L Y)$ is a weak homotopy equivalence. In the commutative diagram

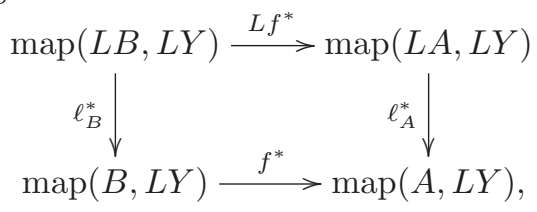

the vertical arrows are weak homotopy equivalences by Lemma 4.2 and the top arrow is also a weak homotopy equivalence because $L f$ is a weak equivalence. Hence, the bottom map has to be a weak homotopy equivalence.

If $f: A \rightarrow B$ is such that $\operatorname{map}(f, X)$ is a weak homotopy equivalence for each $L$-local object $X$, then, using Lemma 4.2 , we deduce that $\operatorname{map}(L f, L A)$ and $\operatorname{map}(L f, L B)$ are weak homotopy equivalences. Hence, $L f$ must be a weak equivalence by [Hir03, Proposition 17.7.6].

Finally, let $X$ be fibrant and such that $\operatorname{map}(f, X)$ is a weak homotopy equivalence for each $L$-equivalence $f$. In particular, $\operatorname{map}\left(\ell_{X}, X\right)$ is a weak homotopy equivalence. On the other hand, $\operatorname{map}\left(\ell_{X}, L X\right)$ is a weak homotopy equivalence by Lemma 4.2. Hence, $\ell_{X}: X \rightarrow L X$ must be a weak equivalence by [Hir03, Proposition 17.7.6].

In what follows, we specialize to combinatorial model categories, i.e., cofibrantly generated model categories whose underlying category is locally presentable. We refer to [Dug01] or [Bar10] for the definition and properties. In a left proper combinatorial model category left Bousfield localizations with respect to a set always exist (cf. [Bar10, Theorem 4.7]). The analogue for cellular model categories is proved in [Hir03, Theorem 4.1.1]. 
The next two results correspond to [CC06, Lemma 1.2] and [CC06, Lemma 1.3], but we drop the hypothesis that the model category be simplicial.

Lemma 4.4. Let $\mathcal{M}$ be a combinatorial model category. Then there is regular cardinal $\mu$ such that, for every class of objects $\mathcal{D}$ in $\mathcal{M}$, the class of $\mathcal{D}$-equivalences $\mathcal{D}^{h \perp}$ is closed under $\mu$-filtered colimits.

Proof. Since map $(-,-)$ is homotopy invariant, we can assume that each object in $\mathcal{D}$ is fibrant. Since we are assuming that $\mathcal{M}$ is combinatorial, there is a regular cardinal $\mu$ such that weak equivalences are preserved by $\mu$-filtered colimits and there are cofibrant and fibrant replacement functors that preserve $\mu$-filtered colimits (cf. [Dug01, Proposition 2.3]). Let $f_{i}: X_{i} \rightarrow Y_{i}$ be $\mathcal{D}$-equivalences for all $i \in I$, where $I$ is a $\mu$-filtered category. Since we are assuming that cofibrant replacement preserves $\mu$-filtered colimits, we can assume that $X_{i}$ and $Y_{i}$ are cofibrant for all $i \in I$.

We have a commutative diagram

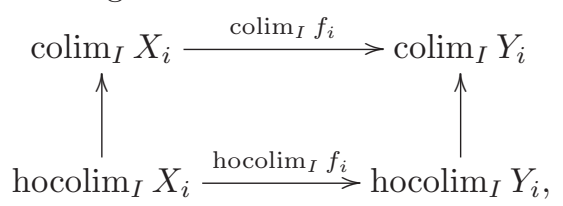

where the vertical arrows are weak equivalences since $\mu$-filtered colimits are homotopy colimits, due to the fact that $\mu$-filtered colimits of weak equivalences are weak equivalences. To finish the proof it is enough to prove that the bottom arrow is a $\mathcal{D}$-equivalence. But now, for every object $Z \in \mathcal{D}$, we have a commutative square

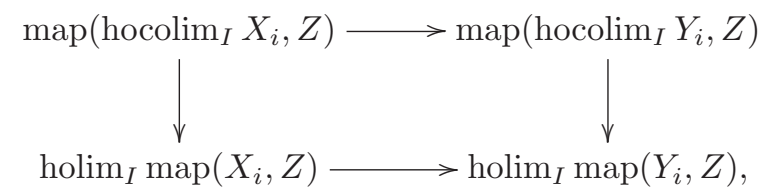

where the vertical arrows are weak homotopy equivalences by [Hir03, Theorem 19.4.4], and the bottom arrow is a weak homotopy equivalence since every $f_{i}$ is a $\mathcal{D}$-equivalence. This proves that hocolim $I f_{i}$ is a $\mathcal{D}$-equivalence.

In the following statement we will need to assume Vopernka's principle. This is a set-theoretical axiom equivalent to the fact that every full subcategory of a locally presentable category that is closed under colimits is a locally presentable coreflective subcategory, i.e., the inclusion has a right adjoint (cf. [AR94, Theorem 6.28 and Theorem 6.29]).

Lemma 4.5. Assume that Vopěnka's principle holds. Let $\mathcal{M}$ be a combinatorial model category and let $\mathcal{D}$ be any class of objects in $\mathcal{M}$. Then there is a set of morphisms $S$ such that the class of $S^{h \perp}$-equivalences, $\left(S^{h \perp}\right)^{h \perp}$, is equal to the class of $\mathcal{D}$-equivalences, $\mathcal{D}^{h \perp}$. Hence, $\left(\mathcal{D}^{h \perp}\right)^{h \perp}$ equals the class of $S$-locals.

Proof. By Lemma 4.4, there is a regular cardinal $\mu^{\prime}$ such that, for every class of objects $\mathcal{E}$ in $\mathcal{M}$ the class of $\mathcal{E}$-equivalences $\mathcal{E}^{h \perp}$ is closed under $\mu^{\prime}$-filtered colimits. On the other hand, $\mathcal{M}$ is $\lambda$-presentable for some regular cardinal $\lambda$ and so is the category of arrows of $\mathcal{M}$ [AR94, Corollary 1.54]. Since we are under Vopěnka's principle, by [AR94, Theorem 6.24 and Theorem 6.29] there exists a regular cardinal 
$\lambda^{\prime}$ and a set of $\lambda^{\prime}$-presentable $\mathcal{D}$-equivalences $S^{\prime}$ such that every morphism in $\mathcal{D}^{h \perp}$ is a $\lambda^{\prime}$-filtered colimit of morphisms in $S^{\prime}$. It then follows that there exists a cardinal $\mu \geqslant \max \left\{\lambda^{\prime}, \mu^{\prime}\right\}$ and a set of $\mathcal{D}$-equivalences $S$ such that every morphism in $\mathcal{D}^{h \perp}$ is a $\mu$-filtered colimit of morphisms in $S$ and $\mathcal{D}^{h \perp}$ is closed under $\mu$-filtered colimits [AR94, Corollary 2.14].

Since every object in $\mathcal{D}$ is $S$-local, every $S$-equivalence is in $\mathcal{D}^{h \perp}$. Conversely, every $g$ in $\mathcal{D}^{h \perp}$ is a $\mu$-filtered colimit of morphisms in $S$. But now $S \subset\left(S^{h \perp}\right)^{h \perp}$ and $\left(S^{h \perp}\right)^{h \perp}$ is closed under $\mu^{\prime}$-filtered colimits by the first comment in the proof. In particular, $\left(S^{h \perp}\right)^{h \perp}$ is also closed under $\mu$-filtered colimits. This implies that $g$ is in $\left(S^{h \perp}\right)^{h \perp}$.

As a direct consequence of Lemma 4.5, we obtain an alternative proof of [CC06, Theorem 2.1] that avoids the assumption of the model category being simplicial. A different proof was given in [RT03, Theorem 2.3].

Corollary 4.6. Assume that Vopěnka's principle holds. Let $\mathcal{M}$ be a left proper combinatorial model category. Then the left Bousfield localization with respect to any class of morphisms $\mathcal{S}$ in $\mathcal{M}$ exists.

Proof. By Lemma 4.5, the class $\mathcal{S}^{h \perp}$ coincides with the class $D^{h \perp}$ with respect to a set of morphisms D. By [Bar10, Theorem 4.7], the Bousfield localization $L_{D} \mathcal{M}$ with respect to $D$ exists in $\mathcal{M}$. Since the $D^{h \perp}$-equivalences coincide with the $\mathcal{S}^{h \perp}$-equivalences, $L_{D} \mathcal{M}$ is also the left Bousfield localization with respect to $\mathcal{S}$.

As noticed in [CC06], in general we cannot take $S$ in the conclusion of Lemma 4.5 to consist of a single morphism. However, it is possible to reduce $S$ to a single morphism if we assume, for instance, that we work in a pointed category. In particular, the next result applies to stable combinatorial model categories.

Corollary 4.7. Assume that Vopěnka's principle holds. Let $\mathcal{M}$ be a pointed combinatorial model category, and let $\mathcal{D}$ be any class of objects in $\mathcal{M}$. Then there is a morphism $f$ such that the class of $f$-equivalences is equal to the class of $\mathcal{D}$-equivalences.

Proof. Let $S$ be the set of morphisms and $\mu$ the regular cardinal as in the proof of Lemma 4.5, and let $f=\coprod s$ for all $s: A \rightarrow B$ in $S$. It is enough to prove that $S^{h \perp}=f^{h \perp}$. If $X$ is an $S$-local object, then every component in the product

$$
\prod \operatorname{map}(s, X) \simeq \operatorname{map}(\coprod s, X)=\operatorname{map}(f, X)
$$

is a weak homotopy equivalence. Hence, $X$ is $f$-local.

Conversely, if $X$ is $f$-local, then $\prod \operatorname{map}(s, X)$ is a weak homotopy equivalence. Since $\mathcal{M}$ is pointed, for each $s$ in $S$ there is a retraction map $r$ such that the composition

$$
\operatorname{map}(s, X) \stackrel{r}{\longrightarrow} \operatorname{map}(\amalg s, X) \simeq \prod \operatorname{map}(s, X) \longrightarrow \operatorname{map}(s, X)
$$

is the identity. Hence, $\operatorname{map}(s, X)$ is a weak homotopy equivalence for each $s$ in $S$. Thus, $X$ is $S$-local.

The following result is a direct consequence of Proposition 4.3 and Lemma 4.5.

Theorem 4.8. Assume that Vopěnka's principle holds. Let $\mathcal{M}$ be a combinatorial model category. If $(L, \ell)$ is any homotopy idempotent functor on $\mathcal{M}$, then there is a set of morphisms $S$ such that the class of $S$-local objects coincides with the class 
of L-local objects. Furthermore, if $\mathcal{M}$ is pointed, then we can take $S$ to consist of a single morphism.

Proof. Let $\mathcal{D}$ be the class of $L$-local objects. It follows from Proposition 4.3 that the class of $\mathcal{D}$-equivalences coincides with the class of $L$-equivalences. Then Lemma 4.5 and Corollary 4.7 finish the proof.

We next extend Theorem 4.8 to any cofibrantly generated model category, in particular, to any cellular model category [Hir03, Definition 12.1.1].

We remind the reader that a Quillen pair $F: \mathcal{N} \rightleftarrows \mathcal{M}: G$ is homotopically surjective if, for every fibrant object $X$ in $\mathcal{M}$ and every cofibrant replacement $(G X)^{c}$ of $G X$, the induced morphism $F(G X)^{c} \rightarrow X$ is a weak equivalence [Dug01, Definition 3.1].

Proposition 4.9. Assume that Vopěnka's principle holds. Let $F: \mathcal{N} \rightleftarrows \mathcal{M}: G$ be a homotopically surjective Quillen pair, and let $\mathcal{N}$ be combinatorial. If $(L, \ell)$ is a homotopy idempotent functor on $\mathcal{M}$, then there is a set of morphisms $S$ in $\mathcal{M}$ such that the class of $S$-local objects coincides with the class of L-local objects. Furthermore, if $\mathcal{M}$ is pointed, then we can take $S$ to consist of a single morphism.

Proof. Let $\mathcal{D}$ be the class of objects of the form $G X$ with $X L$-local. Notice that they are fibrant because $G$ preserves fibrant objects. By Lemma 4.5 , there is a set of morphisms $S^{\prime}$ in $\mathcal{N}$ such that the class of $S^{\prime}$-locals coincide with $\left(\mathcal{D}^{h \perp}\right)^{h \perp}$. Let $S=\left\{F f^{c} \mid f \in S^{\prime}\right\}$. We claim that the $L$-locals coincide with the $S$-locals.

Let $X$ be $L$-local (thus fibrant). By hypothesis, the morphism $F(G X)^{c} \rightarrow X$ is a weak equivalence. By definition, $G X$ is $S^{\prime}$-local. Hence,

$$
\operatorname{map}(f, G X) \simeq \operatorname{map}\left(f, G\left(F(G X)^{c}\right)^{f}\right) \simeq \operatorname{map}\left(F\left(f^{c}\right), F(G X)^{c}\right)
$$

are weak homotopy equivalences for any $f$ in $S^{\prime}$. In particular, $X \simeq F(G X)^{c}$ is $S$-local.

Now let $X$ be $S$-local. By definition, $\operatorname{map}\left(F f^{c}, X\right) \simeq \operatorname{map}(f, G X)$ are weak homotopy equivalences for every $f$ in $S^{\prime}$. Hence, $G X$ is $S^{\prime}$-local; i.e., $G X$ is in $\left(\mathcal{D}^{h \perp}\right)^{h \perp}$.

By Proposition 4.3, to prove that $G X$ is $L$-local it is enough to prove that, for every $L$-equivalence $g, \operatorname{map}(g, G X) \simeq \operatorname{map}\left(F g^{c}, X\right)$ is a weak equivalence. Since we have already proved that $G X$ is $D^{h \perp}$-local, the proof will be finished if we can show that $g$ is a $\mathcal{D}$-equivalence if and only if $F g^{c}$ is an $L$-equivalence. But, by Proposition 4.3 again, both conditions are equivalent to the fact that $\operatorname{map}(g, G Y) \simeq \operatorname{map}\left(F g^{c}, Y\right)$ is a weak equivalence for all $L$-local objects $Y$.

The following result generalizes [CC06, Theorem 2.3] to cofibrantly generated model categories that are not necessarily locally presentable nor simplicial. It also gives a positive answer to a question by Farjoun in [Far96] for a broad family of model categories.

Corollary 4.10. Assume that Vopěnka's principle holds. Let $\mathcal{M}$ be a cofibrantly generated model category. If $(L, \ell)$ is a homotopy idempotent functor on $\mathcal{M}$, then there is a set of morphisms $S$ such that the class of $S$-local objects coincides with the class of L-local objects. Furthermore, if $\mathcal{M}$ is pointed, then we can take $S$ to consist of a single morphism.

Proof. Since we are assuming Vopěnka's principle, [Rap09, Theorem 1.1] implies that there is a Quillen equivalence (in particular, homotopically surjective) $\mathcal{N} \rightleftarrows \mathcal{M}$ where $\mathcal{N}$ is combinatorial. Hence, the result follows from Proposition 4.9. 
The cofibrantly generated condition in Corollary 4.10 is necessary. In [Cho05] an example is given of a left Bousfield localization with respect to a class of morphisms in a (non cofibrantly generated) model category that cannot be a left Bousfield localization with respect to any set.

We next state the analogues of the main results in this section but for augmented homotopy idempotent functors. We omit the proofs since they are easily reproduced following the proofs for the coaugmented case. An augmented homotopy idempotent functor in a model category $\mathcal{M}$ is a functor $C: \mathcal{M} \rightarrow \mathcal{M}$ together with a natural transformation $\varepsilon: C \rightarrow 1$ such that $C$ sends weak equivalences to weak equivalences and the natural morphisms $\varepsilon_{C X}, C \varepsilon_{X}: C C X \rightarrow C X$ are equal in the homotopy category $\mathrm{Ho}(\mathcal{M})$ and both are weak equivalences for every object $X$ in $\mathcal{M}$. The following result generalizes [Cho07, Theorem 1.4] to combinatorial model categories not necessarily simplicial.

Corollary 4.11. Assume that Vopěnka's principle holds. Let $\mathcal{M}$ be a right proper combinatorial model category. Then the right Bousfield localization with respect to any class of objects in $\mathcal{M}$ exists.

The following result generalizes [Cho07, Theorem 2.1] to cofibrantly generated model categories not necessarily locally presentable nor simplicial.

Theorem 4.12. Assume that Vopěnka's principle holds. Let $\mathcal{M}$ be a cofibrantly generated model category. If $(C, \varepsilon)$ is a homotopy augmented idempotent functor on $\mathcal{M}$, then there is a set of objects $D$ such that the class of $D$-cellular equivalences coincides with the class of $C$-cellular equivalences. Furthermore, if $\mathcal{M}$ is pointed, then we can take $D$ to consist of a single object.

\section{References}

[Ada75] J.F. Adams, Localisation and completion, Department of Mathematics, University of Chicago, Chicago, Ill., 1975.

[AR94] J. Adámek and J. Rosický, Locally presentable and accessible categories, London Mathematical Society Lecture Note Series, vol. 189, Cambridge University Press, Cambridge, 1994.

[Bar10] C. Barwick, On left and right model categories and left and right Bousfield localizations, Homology, Homotopy Appl. 12 (2010), no. 2, 245-320.

[Bor94] F. Borceux, Handbook of categorical algebra. 2, Encyclopedia of Mathematics and its Applications, vol. 51, Cambridge University Press, Cambridge, 1994, Categories and structures.

[CC06] C. Casacuberta and B. Chorny, The orthogonal subcategory problem in homotopy theory, An alpine anthology of homotopy theory, Contemp. Math., vol. 399, Amer. Math. Soc., Providence, RI, 2006, 41-53.

[CRT] C. Casacuberta, O. Raventós and A. Tonks, Comparing localizations across adjunctions, arXiv:1404.7340.

[CSS05] C. Casacuberta, D. Scevenels and J.H. Smith, Implications of largecardinal principles in homotopical localization, Adv. Math. 197 (2005), no. $1,120-139$. 
[Cho05] B. Chorny, Localization with respect to a class of maps. II. Equivariant cellularization and its application, Israel J. Math. 147 (2005), 141-155.

[Cho07] _ Abstract cellularization as a cellularization with respect to a set of objects, Categories in algebra, geometry and mathematical physics, Contemp. Math., vol. 431, Amer. Math. Soc., Providence, RI, 2007, $165-170$.

[DHKS04] W.G. Dwyer, P.S. Hirschhorn, D.M. Kan and J.H. Smith, Homotopy limit functors on model categories and homotopical categories, Mathematical Surveys and Monographs, vol. 113, American Mathematical Society, Providence, RI, 2004.

[DK80a] W.G. Dwyer and D.M. Kan, Calculating simplicial localizations, J. Pure Appl. Algebra 18 (1980), no. 1, 17-35.

[DK80b] _ Function complexes in homotopical algebra, Topology 19 (1980), no. 4, 427-440.

[DK80c] S S Simplicial localizations of categories, J. Pure Appl. Algebra 17 (1980), no. 3, 267-284.

[Dug01] D. Dugger, Combinatorial model categories have presentations, Adv. Math. 164 (2001), no. 1, 177-201.

[Far96] E.D. Farjoun, Cellular spaces, null spaces and homotopy localization, Lecture Notes in Mathematics, vol. 1622, Springer-Verlag, Berlin, 1996.

[Far96b] Higher homotopies of natural constructions, J. Pure Appl. Algebra 108 (1996), no. 1, 23-34.

[Hir03] P.S. Hirschhorn, Model categories and their localizations, Mathematical Surveys and Monographs, vol. 99, American Mathematical Society, Providence, RI, 2003.

[Kel82] G.M. Kelly, Basic concepts of enriched category theory, London Mathematical Society Lecture Note Series, vol. 64, Cambridge University Press, Cambridge-New York, 1982.

[Qui73] D. Quillen, Higher algebraic K-theory. I, Algebraic K-theory, I: Higher $K$-theories (Proc. Conf., Battelle Memorial Inst., Seattle, Wash., 1972), Lecture Notes in Math., vol. 341, Springer, Berlin, 1973.

[Rap09] G. Raptis, On the cofibrant generation of model categories, J. Homotopy Relat. Struct. 4 (2009), no. 1, 245-253.

[RT03] J. Rosický and W. Tholen, Left-determined model categories and universal homotopy theories, Trans. Amer. Math. Soc. 355 (2003), no. 9, 3611-3623.

Oriol Raventós oriol.raventos-morera@ur.de

Fakultät für Mathematik, Universität Regensburg, 93040 Regensburg, Germany 